\title{
Desnutrición hospitalaria y variables antropométricas para la valoración nutricional.
}

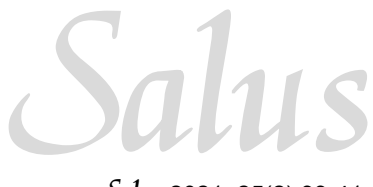

Hospital malnutrition and anthropometric variables for nutritional assessment.

Salus 2021; 25(2):33-41.

https://doi.org/10.54139/salus.v25i2.60

María Laura Claramonte Larghi'1,2 (D), Luis Pérez Ybarra ${ }^{3} \mathbb{D}$, Nirza Noguera Machado ${ }^{3}$ (D) , Luis Edgardo Ojeda ${ }^{4}(\mathbb{D}$

\section{RESUMEN}

Introducción: La desnutrición hospitalaria comprende toda situación carencial, sea causa de la enfermedad, ingesta inadecuada de nutrientes, procedimientos terapéuticos, hospitalización y sus complicaciones, siendo la causa más frecuente del aumento de la tasa de morbi-mortalidad, afectando de forma especial al paciente hospitalizado con una prevalencia del 40-60\%. Objetivo: Caracterizar la evaluación nutricional antropométrica en el paciente hospitalizado, mediante indicadores de dimensión corporal: peso, talla, índice de masa corporal y porcentaje de pérdida de peso en el tiempo para detectar desnutrición hospitalaria. Metodología: Se estudiaron 103 pacientes adultos ingresados por los Departamentos de Medicina interna, Traumatología y Cirugía maxilofacial, quienes previa autorización fueron pesados, tallados tanto al ingreso como al egreso de su hospitalización. Se calculó pérdida de peso, pérdida de índice de masa corporal (IMC) y tasa de pérdida de peso durante la hospitalización. Resultados: el IMC discriminado por sexo y edad detectó más desnutrición ( $39 \%$ vs $12 \%$ ). Durante la hospitalización $95 \%$ de los pacientes presentaron pérdida de peso (88\% severa, $4 \%$ moderada), siendo estadísticamente significativa $(p \leq 0,05)$ por grupo etario, departamento, dieta y días de hospitalización. La prevalencia de desnutrición hospitalaria al egreso fue de $92 \%$. Conclusión: EI IMC como único indicador antropométrico en el paciente hospitalizado pierde capacidad para detectar desnutrición hospitalaria. La diferencia de peso (\%) en función del tiempo,

Departamento de Fisiopatología y Farmacología, Escuela de Medicina sede Aragua, Facultad de Ciencias de la Salud, Universidad de Carabobo, MaracayVenezuela.

2 División de asuntos Médicos del Hospital Militar "Coronel Elbano Paredes Vivas", Maracay-Venezuela.

3 Departamento de Ciencias Básicas, Escuela de Bioanálisis sede Aragua, Facultad de Ciencias de la Salud, Universidad de Carabobo, Maracay-Venezuela

${ }^{4}$ Departamento de Fisiología y Bioquímica, Escuela de Medicina sede Aragua, Facultad de Ciencias de la Salud, Universidad de Carabobo, Maracay-Venezuela.

Autor de correspondencia: Luis Pérez Ybarra (D)

E-mail: Lmpy2005@gmail.com

Recibido: 13-07-2020

Aprobado: 10-09-2021 demostró ser más acertada en el paciente hospitalizado, ya que permite detectar desnutrición aguda al captar con mayor exactitud los cambios involuntarios de peso. Al combinar ambos indicadores antropométricos la prevalencia de desnutrición hospitalaria fue del $93 \%$.

Palabras clave: valoración nutricional, desnutrición hospitalaria, pérdida de peso, índice de masa corporal, indicadores antropométricos.

\section{ABSTRACT}

Introduction: Hospital malnutrition includes any deficiency situation, whether it is the cause of the disease, inadequate intake of nutrients, therapeutic procedures, hospitalization and its complications, being the most frequent cause of the increase in the morbidity and mortality rate, especially affecting the hospitalized patient with a prevalence of $40-60 \%$. Objective: To characterize the anthropometric nutritional evaluation in the hospitalized patient, by means of indicators of body dimension: weight, height, body mass index and percentage of weight loss over time to detect hospital malnutrition. Methodology: 103 adult patients admitted by the Departments of Internal Medicine, Traumatology and Maxillofacial Surgery were studied, who, with prior authorization, were weighed, sized both on admission and on discharge from hospitalization. Weight loss, body mass index loss $\mathrm{BMI}$, and weight loss rate were calculated during hospitalization. Results: the BMI discriminated by sex and age detected more malnutrition (39\% vs $12 \%$ ). During hospitalization, $95 \%$ of the patients presented weight loss ( $88 \%$ severe, $4 \%$ moderate), being statistically significant $(\mathrm{p} \leq 0.05)$ by age group, department, diet, and days of hospitalization. The prevalence of hospital malnutrition at discharge was $92 \%$. Conclusion: BMI as the only anthropometric indicator in hospitalized patients loses the ability to detect hospital malnutrition. The difference in weight (\%) as a function of time, proved to be more accurate in hospitalized patients, since it allows detecting acute malnutrition by capturing involuntary changes in weight with greater accuracy. When combining both anthropometric indicators, the prevalence of hospital malnutrition was $93 \%$.

Key words: nutritional assessment, hospital malnutrition, weight loss, body mass index, anthropometric indicators.

\section{INTRODUCCIÓN}

El deterioro del estado nutricional de los pacientes hospitalizados es multifactorial (1), ocasionando disminución de la resistencia a la infección y sepsis, retraso de la cicatrización de heridas, estancia hospitalaria prolongada, 
aumento de recursos dedicados a la atención y tratamiento de complicaciones asociadas, riesgo de re-admisiones y mortalidad (2).

La desnutrición no solo influye negativamente en el área física y cognitiva. Se ha evidenciado compromiso del sistema inmune, aumento de la susceptibilidad a enfermedades (transmisibles y no transmisibles) (3), sumado a que representa una pesada carga con consecuencias sociales y económicas negativas para las personas, familias, comunidades y Estados $(4,5)$.

La prevalencia de la desnutrición hospitalaria oscila entre 40 a $60 \%$, dependiendo de la población estudiada y los criterios y métodos utilizados para realizar el diagnóstico (6). Salvador-Monferrer et al. realizaron una revisión de 117 trabajos, encontrando mucha variabilidad en los métodos utilizados, los criterios diagnósticos empleados para definir desnutrición y amplias diferencias en las variables analizadas (grupo etario, patología, servicio, etc.) (7).

No siempre pueden compararse las prevalencias que puede diferir significativamente según el método empleado (Valoración Global Subjetiva (VGS), Malnutrition Universal Screening Tool (MUST), Mini Nutritional Assessment (MINA), variables antropométricas, impedancia, entre otros) (7). Gomes-Beghetto et al. encontraron que el diagnóstico de desnutrición basado en IMC subestima la prevalencia, mientras que se sobreestima al utilizar VGS (8). Los autores de esta amplia revisión concluyen que hace falta definir las herramientas necesarias para una evaluación nutricional oportuna, y efectiva al momento de identificar un paciente con riesgo de desnutrición (7).

La mayoría de los trabajos conocidos sobre desnutrición hospitalaria han sido realizados en países desarrollados (9), donde el factor socio económico del paciente y de la institución no repercute sobre la condición nutricional. En países en vías de desarrollo el problema del déficit nutricional puede ser aún más grave, si se tiene en cuenta que en estos países el factor socioeconómico influye en la elevada prevalencia de la desnutrición (10).

Ortiz, en 2013 evaluó la desnutrición en adultos mayores institucionalizados utilizando la escala MINA, encontrando una prevalencia de desnutrición del $53,85 \%$, siendo mayor en el sexo femenino y grupo etario de 60-70 años (11). Perez-Flores et al., utilizaron VGS para identificar pacientes con desnutrición al momento de la hospitalización $(25,2 \%)$, representando un factor de riesgo esta condición durante su hospitalización, aumentando la morbimortalidad en ellos (12). Vazquez et al. hicieron seguimiento durante su hospitalización de los pacientes catalogados por la VGS con algún grado de desnutrición $(83,8 \%)$, encontrando que menos del $10 \%$ se le realizó una evaluación nutricional integrada y menos del $30 \%$ recibio terapia nutricional (13).

Botina et al., concluyeron que la realización de una valoración sistemática al momento del ingreso permite identificar a pacientes con deficiencias nutricionales y el riesgo de desnutrición que requieren de un soporte nutricional precoz (14). Estos trabajos demuestran la importancia que tiene la valoracion nutricional de los pacientes al ingreso, también debe ser importante el monitorear la condición nutricional durante la hospitalización para detectar precozmente la desnutrición e instaurar una terapia nutricional oportuna y eficaz. El índice de masa corporal (IMC) a menudo es un indicador útil en la población adulta y un valor inferior a los 18,5 kg/m2 está asociado con una desnutrición importante $(15,16)$. El hallazgo de una pérdida de peso no intencional reciente es una variable clínica fácil de medir, se correlaciona de manera estrecha con el grado de estrés y es el indicador más sensible de desnutrición aguda durante la hospitalización (15). El déficit funcional comienza a desarrollarse cuando la pérdida de peso supera el $10 \%$, aún en los pacientes obesos; si la pérdida de peso es mayor del $20 \%$, la capacidad de combatir el estrés está disminuida de manera severa $(1,17)$.

En esta investigación se evaluó la condición nutricional de los pacientes ingresados en el Hospital Militar Cnel. Elbano Paredes Vivas que padecen un riesgo biológico agudo mediante variables e indicadores antropométricos, así como el seguimiento nutricional durante su hospitalización para la detección de desnutrición hospitalaria.

\section{MATERIALES Y MÉTODOS}

Estudio prospectivo de corte transversal. Se evaluaron pacientes mayores de 18 años, ingresados por los departamentos de Medicina interna, Traumatología y Cirugía maxilofacial, del Hospital Militar "Cnel. Elbano Paredes Vivas" (HOMELPAVI) ubicado en la ciudad de MaracayVenezuela, durante el período comprendido entre julio-2017 y febrero-2018. Los individuos fueron seleccionados a través de un muestreo no probabilístico de tipo intencional y autorizaron su participación en el estudio mediante la firma de un consentimiento informado. El trabajo contó con la aprobación del comité de bioética del HOMELPAVI con fecha 12 de junio de 2017.

Criterios de inclusión: individuos hospitalizados, de uno $\mathrm{u}$ otro sexo, que deambulan por sus propios pies, con consentimiento del paciente aceptando la realización de las mediciones antropométricas.

Criterios de exclusión: presencia de patologías que modifiquen el peso real (enfermedad renal, insuficiencia 
cardíaca, insuficiencia hepática, enfermedades degenerativas), paciente encamado o conectado a ventilación mecánica.

Recolección de datos: una vez ingresado el paciente, se procedió a realizarles mediciones antropométricas antes de las 48 horas y las mismas se repitieron al egreso. Con ellas se realizó el diagnóstico nutricional de ingreso y egreso. De la historia clínica se tomaron los datos del tipo de dieta indicada y recibida, Los grupos etarios se clasificaron en adultos jóvenes (AJ) de 18 a 39 años, adultos mayores (AM) de 40 a 60 años, y adultos de la tercera edad (ATE) de 61 años en adelante.

Variables biomédicas: edad (años), sexo, causa de hospitalización, patología de base, días de hospitalización y tipo de dieta indicada. Variables antropométricas: talla $(\mathrm{m})$, peso usual (referido por el paciente antes de enfermarse), peso actual (el de ingreso y de egreso) $(\mathrm{kg})$, pérdida de peso (PP) absoluta $(\mathrm{kg})$ y PP relativa $(\%)$, IMC ( $\mathrm{kg} / \mathrm{m} 2)$, tasa de PP (kg/día) en relación con los días de hospitalización. Con estas variables e indicadores se procedió a realizar el diagnóstico nutricional.

Para la valoración del IMC se utilizaron los puntos de corte de la FAO-OMS (16) y los del Laboratorio de Evaluación Nutricional de la Universidad Simón Bolívar (LEN-USB) (15), construidos a partir de las tablas de referencia de Frisancho (18) de percentiles de peso para la talla discriminados por sexo, edad y contextura. Se clasificó en: eutrófico (sin malnutrición), desnutrido, sobrepesado (IMC>25 kg/m2 y $<30 \mathrm{~kg} / \mathrm{m} 2$, ocasionado por aumento de masa muscular o masa grasa), obeso grado II (IMC $\geq 30 \mathrm{~kg} / \mathrm{m} 2$, debido aumento masa grasa) La severidad de la pérdida de peso en relación al tiempo se determinó mediante los puntos de corte de Blackburn et al. en leve, moderada y severa (19).

Análisis estadístico: Se calcularon los estadísticos descriptivos media aritmética $(\bar{x})$, desviación estándar (DS), error estándar de la media (EE) y valores mínimo y máximo, clasificados por sexo, grupo etario, departamento y tipo de dieta asignada para las diferencias entre peso, IMC y tasa de pérdida de peso diaria. Para la clasificación por sexo se aplicó la prueba no paramétrica para dos grupos independientes de Wilcoxon-Mann-Whitney. Para las clasificaciones por grupo etario, departamento y tipo de dieta asignada se aplicó la prueba no paramétrica de Kruskal-Wallis para $\mathrm{k}(\mathrm{k} \geq 3)$ muestras independientes y la correspondiente prueba de comparaciones de medias no paramétrica. Se calcularon las prevalencias para la PP al egreso categorizada en ninguna, leve, moderada y severa.

Para identificar si existe asociación entre el tiempo de hospitalización y las diferencias entre peso, IMC y tasa de pérdida de peso diaria, se calcularon los coeficientes de correlación no paramétricos de rangos de Spearman y se construyeron además los gráficos de dispersión. Para verificar si existe asociación entre la PP al egreso con las variables sexo, grupo etario, departamento de ingreso y dieta administrada, se construyeron las tablas de contingencia correspondientes y sobre éstas se aplicó la prueba de independencia de $\mathrm{x} 2$.

A los diagnósticos nutricionales de egreso e ingreso se aplicó la prueba de rangos con signo de Wilcoxon para verificar si hubo cambio de respuesta significativo al egreso con respecto al ingreso del paciente, para lo cual se reportarán y analizarán las sumas de rangos negativos (SRank ${ }_{(-)}$) y positivos $\left(\right.$ SRank $\left._{(+)}\right)$. Sobre aquellos diagnósticos que resultaron estadísticamente significativos se construyeron los gráficos de barra para las distribuciones de frecuencias absolutas a fin de evidenciar el cambio de respuesta detectado.

El diagnóstico nutricional fue realizado según los criterios de la FAO-OMS y de LEN-USB, se compararon con la prueba de concordancia basada en el coeficiente $\mathrm{k}$ de Cohen y se caracterizó el grado de concordancia encontrado según el criterio sugerido por López y Pita (20).

El nivel se significación se fijó en $5 \%$, por lo que un resultado se consideró estadísticamente significativo si $p \leq 0,05$. Los datos se procesaron utilizando los programas estadísticos Minitab 18.0 y Statistix 9.0 para Windows.

\section{RESULTADOS}

La muestra estuvo conformada por 103 pacientes adultos ingresados por los departamentos de Medicina interna $(60,2 \%)$, Traumatología $(30,1 \%)$ y Cirugía maxilofacial $(9,7 \%)$. La edad promedio fue de $\bar{x}=44,8 \pm 20,6$ años, distribuidos AJ (45,6\%), AM (31,1\%) y ATE (23,3\%). El $32 \%$ de los pacientes eran del sexo femenino. Los días de hospitalización oscilaron entre 4 y 30, $\bar{x}=13,4 \pm 6,4$ días.

Se registraron como causas de hospitalización en el departamento de medicina: patologías cardiovasculares $(15,5 \%)$, enfermedades infecciosas (14,6\%), evento cerebro-vascular isquémico $(8,7 \%)$, hematológicas $(5,8 \%)$, otras causas $(15,9 \%)$ mientras que en los departamentos quirúrgicos: corrección fractura de miembro superior $(23 \%)$, cirugía por otras causas en miembro superior $(4,9 \%)$, limpieza quirúrgica miembro superior $(1,9 \%)$ y corrección quirúrgica de fractura maxilofacial $(9,7 \%)$. Se indicó dieta al $89,4 \%$ de los pacientes (70,9\% completa, 9,7\% licuada, $4,9 \%$ líquida y $3,9 \%$ blanda). El 10,6\% recibió dieta absoluta. Los estadísticos descriptivos relativos a las variables biomédicas, antropométricas y tiempo de hospitalización se presentan en la Tabla 1.

\section{Salus Revista de la facultad de ciencias de la Salud. Universidad de Garahoho. Mayo/Agosto 2021 Vol. 25 № 2}


Tabla 1. Estadísticos descriptivos para las variables biomédicas, antropométricas y estancia hospitalaria clasificados por sexo

\begin{tabular}{|c|c|c|c|c|c|c|c|}
\hline Variable & Sexo & $\mathbf{N}$ & Media & DS & EE & Mínimo & Máximo \\
\hline \multirow[t]{2}{*}{ Edad (años) } & $\mathrm{F}$ & 33 & 51,33 & 20,63 & 3,59 & 18 & 89 \\
\hline & $\mathrm{M}$ & 70 & 41,73 & 19,97 & 2,39 & 19 & 90 \\
\hline \multirow{2}{*}{$\begin{array}{l}\text { Días de } \\
\text { hospitalización }\end{array}$} & $F$ & 33 & 11,49 & 5,64 & 0,98 & 5 & 30 \\
\hline & $\mathrm{M}$ & 70 & 14,30 & 6,61 & 0,79 & 4 & 30 \\
\hline \multirow[t]{2}{*}{ Talla (m) } & $\mathrm{F}$ & 33 & 1,56 & 0,06 & 0,01 & 1,43 & 1,7 \\
\hline & $M$ & 70 & 1,69 & 0,07 & 0,01 & 1,52 & 1,87 \\
\hline \multirow{2}{*}{$\begin{array}{l}\text { Peso usual } \\
(\mathrm{kg})\end{array}$} & $F$ & 33 & 61,61 & 12,53 & 2,18 & 45 & 104 \\
\hline & M & 70 & 76,36 & 11,86 & 1,42 & 50 & 105 \\
\hline \multirow{2}{*}{$\begin{array}{l}\text { Peso al } \\
\text { ingreso }(\mathrm{kg})\end{array}$} & $\mathrm{F}$ & 33 & 58,10 & 10,72 & 1,87 & 43,2 & 94 \\
\hline & M & 70 & 74,01 & 12,89 & 1,54 & 48 & 105 \\
\hline \multirow{2}{*}{$\begin{array}{l}\text { Peso al egreso } \\
(\mathrm{kg})\end{array}$} & $F$ & 33 & 54,24 & 9,82 & 1,71 & 40 & 82,6 \\
\hline & $M$ & 70 & 70,31 & 12,77 & 1,53 & 44 & 98,1 \\
\hline \multirow{2}{*}{$\begin{array}{l}\text { IMC al ingreso } \\
(\mathrm{kg} / \mathrm{m} 2)\end{array}$} & $F$ & 33 & 23,67 & 3,65 & 0,64 & 18,45 & 33,3 \\
\hline & M & 70 & 25,77 & 3,73 & 0,45 & 19,11 & 35,99 \\
\hline \multirow{2}{*}{$\begin{array}{l}\text { IMC al egreso } \\
(\mathrm{kg} / \mathrm{m} 2)\end{array}$} & $F$ & 33 & 22,11 & 3,40 & 0,59 & 16,85 & 29,27 \\
\hline & $M$ & 70 & 24,47 & 3,65 & 0,44 & 17,92 & 33,84 \\
\hline
\end{tabular}

Tabla 2. Diferencias de peso, IMC y tasa de pérdida de peso distribuidas por sexo. Pacientes Adultos Hospitalizados.

\begin{tabular}{|c|c|c|c|c|c|c|c|c|}
\hline Variable & Sexo & $\mathbf{N}$ & Media & DS & EE & Mínimo & Máximo & p \\
\hline \multirow{2}{*}{$\begin{array}{l}\text { Pérdida de peso } \\
\text { hospitalización }\end{array}$} & $\mathrm{F}$ & 33 & $-3,86$ & 2,84 & 0,49 & -12 & 0 & 0,6533 \\
\hline & M & 70 & $-3,70$ & 1,78 & 0,21 & $-7,3$ & 0 & \\
\hline \multirow{2}{*}{$\begin{array}{l}\text { Pérdida de peso } \\
\text { hospitalización (\%) }\end{array}$} & $\mathrm{F}$ & 33 & $-6,54$ & 4,12 & 0,72 & $-17,65$ & 0 & 0,0755 \\
\hline & M & 70 & $-5,11$ & 2,54 & 0,30 & $-10,45$ & 0 & \\
\hline \multirow[t]{2}{*}{ Diferencia de IMC } & $\mathrm{F}$ & 33 & $-1,57$ & 1,12 & 0,20 & $-4,99$ & 0 & 0,4666 \\
\hline & M & 70 & $-1,31$ & 0,65 & 0,08 & $-2,9$ & 0 & \\
\hline \multirow{2}{*}{$\begin{array}{l}\text { Diferencia de IMC } \\
(\%)\end{array}$} & $\mathrm{F}$ & 33 & $-6,54$ & 4,11 & 0,72 & $-17,63$ & 0 & 0,0732 \\
\hline & $M$ & 70 & $-5,11$ & 2,54 & 0,30 & $-10,46$ & 0 & \\
\hline \multirow{2}{*}{$\begin{array}{l}\text { Diferencia de peso } \\
\text { al ingreso }\end{array}$} & $\mathrm{F}$ & 33 & $-3,51$ & 5,63 & 0,98 & $-15,5$ & 10 & 0,0740 \\
\hline & $M$ & 70 & $-2,34$ & 6,08 & 0,73 & -20 & 17 & \\
\hline \multirow{2}{*}{$\begin{array}{l}\text { Diferencia de peso } \\
\text { al ingreso }(\%)\end{array}$} & $\mathrm{F}$ & 33 & $-5,09$ & 8,56 & 1,49 & $-19,38$ & 18,52 & 0,0547 \\
\hline & $M$ & 70 & $-3,04$ & 8,05 & 0,96 & $-29,41$ & 21,25 & \\
\hline \multirow{2}{*}{$\begin{array}{l}\text { Tasa de pérdida de } \\
\text { peso diaria }\end{array}$} & $\mathrm{F}$ & 33 & $-0,34$ & 0,22 & 0,04 & $-0,8$ & 0 & 0,0724 \\
\hline & $M$ & 70 & $-0,26$ & 0,09 & 0,01 & $-0,46$ & 0 & \\
\hline \multirow[t]{2}{*}{$\begin{array}{l}\text { Tasa de pérdida de } \\
\text { peso diaria }(\%)\end{array}$} & $F$ & 33 & $-0,59$ & 0,36 & 0,06 & $-1,51$ & 0 & $0,0002^{*}$ \\
\hline & $M$ & 70 & $-0,36$ & 0,13 & 0,02 & $-0,7$ & 0 & \\
\hline
\end{tabular}

$\left(^{\star}\right)$ Diferencia estadísticamente significativa al $5 \%$.

Al comparar las variables antropométricas según el grupo etario sólo se detectaron diferencias estadísticamente significativas para \%PP al ingreso $(p<0,0001)$, el cual fue más acentuado en los ATE $(-6,36 \mathrm{~kg})$, seguidos de AM $(-3,23$ $\mathrm{kg}$ ) y en menor medida en AJ $(-0,51 \mathrm{~kg})$.
Según el departamento por el cual ingresaron, se observaron diferencias significativas para \%PP e IMC tanto al ingreso $(p<0,0001)$, (departamento de medicina) como al egreso $(p=0,0008)$, presentando la mayor diferencia en el departamento de traumatología y un comportamiento similar se observó para la diferencia de IMC $(p=0,0069)$. (Tabla 3$)$. 
Tabla 3. Diferencias de peso $(\mathrm{kg})$, IMC $(\mathrm{kg} / \mathrm{m} 2)$ y tasa de pérdida de peso (kg/día) clasificadas por departamento.

\begin{tabular}{|l|c|c|c|c|c|c|c|}
\hline Variable & Servicio & $\mathbf{n}$ & Media & DS & EE & Mínimo & Máximo \\
\hline $\begin{array}{l}\text { Diferencia } \\
\text { de peso }\end{array}$ & MED & 62 & $-4,51 \mathrm{~B}$ & 7,12 & 0,90 & -20 & 17 \\
\hline Usual-Ig & TRA & 31 & $0,00 \mathrm{~A}$ & 0,00 & 0,00 & 0 & 0 \\
\hline & MAX & 10 & $0,00 \mathrm{~A}$ & 0,00 & 0,00 & 0 & 0 \\
\hline $\begin{array}{l}\text { Diferencia } \\
\text { de peso }\end{array}$ & MED & 62 & $-3,47 \mathrm{~A}$ & 2,50 & 0,32 & -12 & 0 \\
\hline Ig-Eg & TRA & 31 & $-4,55 \mathrm{~B}$ & 1,18 & 0,21 & $-6,9$ & $-2,5$ \\
\hline
\end{tabular}

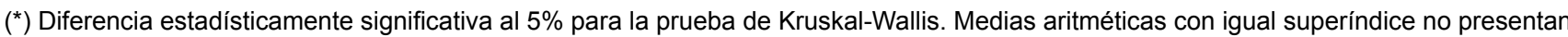
diferencias estadísticamente significativas. MED: medicina interna, TRA: traumatología, MAX: cirugía máxilofacial. Ig: Ingreso. Eg: egreso. IMC: índice de masa corporal.

El coeficiente de correlación de rangos de Spearman indicó que hubo asociación estadísticamente significativa entre los días de hospitalización y las variables diferencia de peso $(r=-0,7427 ; p<0,0001)$ y diferencia de IMC ( $r=-0,7002$; $p<0,0001)$. Los signos negativos de los coeficientes de correlación indican que en la medida que el paciente pasa más tiempo hospitalizado, más acentuada es la pérdida de peso y la disminución del IMC (Fig. 1A y 1B); por otra parte, no se detectó asociación estadísticamente significativa para la variable tasa de pérdida de peso diaria. $(r=0,1024$; $p=0,3029)$, (Fig.1C).

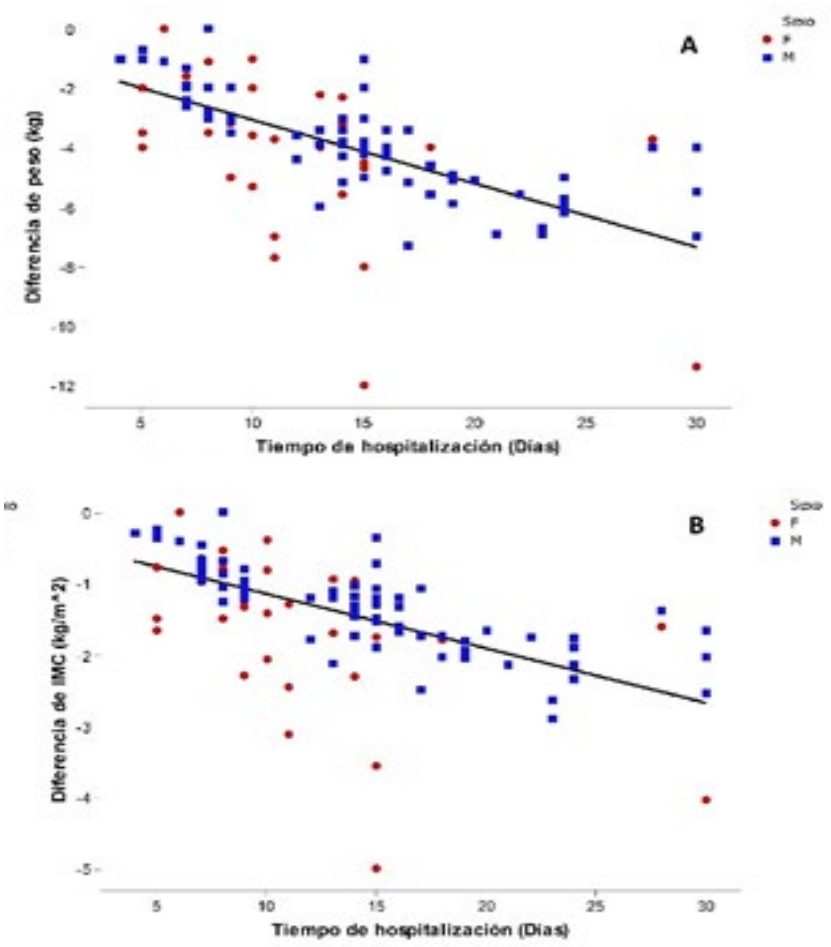

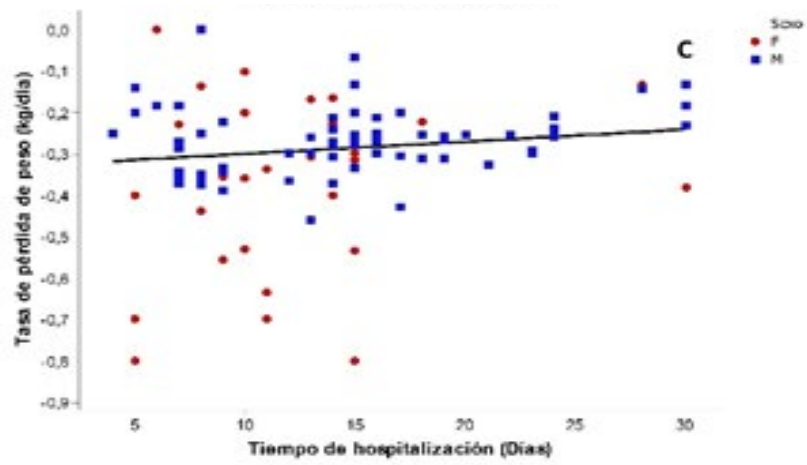

Fig. 1. Gráficos de dispersión y líneas de tendencia durante el tiempo de hospitalización (días). A) Diferencia de peso (kg), B) IMC (kg/m2), C) Tasa de pérdida de peso (kg/día).

Se indicó dieta al $99,4 \%$ de los pacientes $(70,9 \%$ completa, $9,7 \%$ licuada, $4,9 \%$ líquida, 3,9\% blanda y $10,6 \%$ dieta absoluta). Se encontró significancia estadística con $\%$ PP ( $p=0,0372)$ e IMC ( $p=0,0448)$; las diferencias más acentuadas recayeron sobre quienes tenían asignadas dietas completa, blanda y absoluta, no observándose diferencias estadísticamente significativas en la tasa diaria de pérdida de peso $(p=0,4794)$.

La severidad de la pérdida de peso durante la hospitalización fue: leve (3\%), moderada (4\%) y severa (88\%), $5 \%$ no presento modificación en el peso (Fig. 2).

La prueba de independencia de $\mathrm{X} 2$ no mostró asociación estadísticamente significativa con género $(x 2=3,68$; $p=0,3483) ;$ grupo etario $(x 2=10,69 ; p=0,0801)$, ni el departamento $(x 2=8,98 ; p=0,1679)$, pero sí con la dieta recibida por el paciente $(x 2=42,74 ; p=0,0029)$, de tal forma que presentaron PP severa $91,78 \%$ con dieta completa, $100 \%$ con dieta blanda y o licuada, $63,6 \%$ con líquida y $60 \%$ con dieta absoluta. (Fig. 2). 


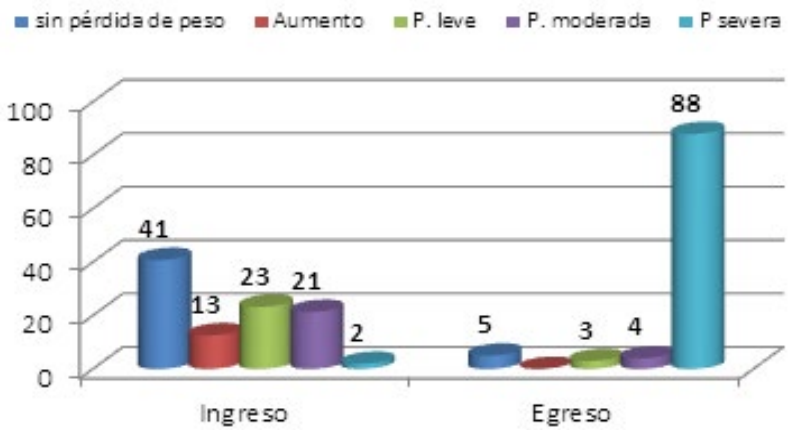

Fig. 2. Clasificación de la variación de peso en relación al tiempo al ingreso y egreso.

Utilizando los puntos de corte de la FAO-OMS para IMC, el diagnóstico nutricional al Ingreso fue: $47,6 \%(49 / 103)$ eutróficos, 41,8\% (43/103) sobrepesados, 9,7\% (10/103) obesos grados II y $1 \%$ (1/103) desnutrido (desnutrición leve); $y$ al Egreso: $51,5 \%$ (53/103) eutróficos, 33,0\% (34/103) sobrepesados, $11,7 \%(12 / 103)$ desnutridos (1\% leve y $10,7 \%$ moderado) y $3,9 \%$ (4/103) obeso grado II, (Fig. 3A).

Con los puntos de corte para IMC de LEN-USB, el diagnóstico nutricional al ingreso fue: $45,6 \%$ (47/103) eutróficos, $14,6 \%$ $(15 / 103)$ obesos grado II, 8,7\% (9/103) sobrepesados y $31,1 \%(32 / 103)$ desnutridos (moderada $21,4 \%$ y leve $9,7 \%)$; y al egreso 46,6\% (48/103) eutróficos, 9,7\% (10/103) sobrepesados, $4,9 \%(5 / 103)$ obesos grado II y desnutridos $38,8 \%(40 / 103)$ (32\% moderada y $6,8 \%$ leve) (Fig. 3B).
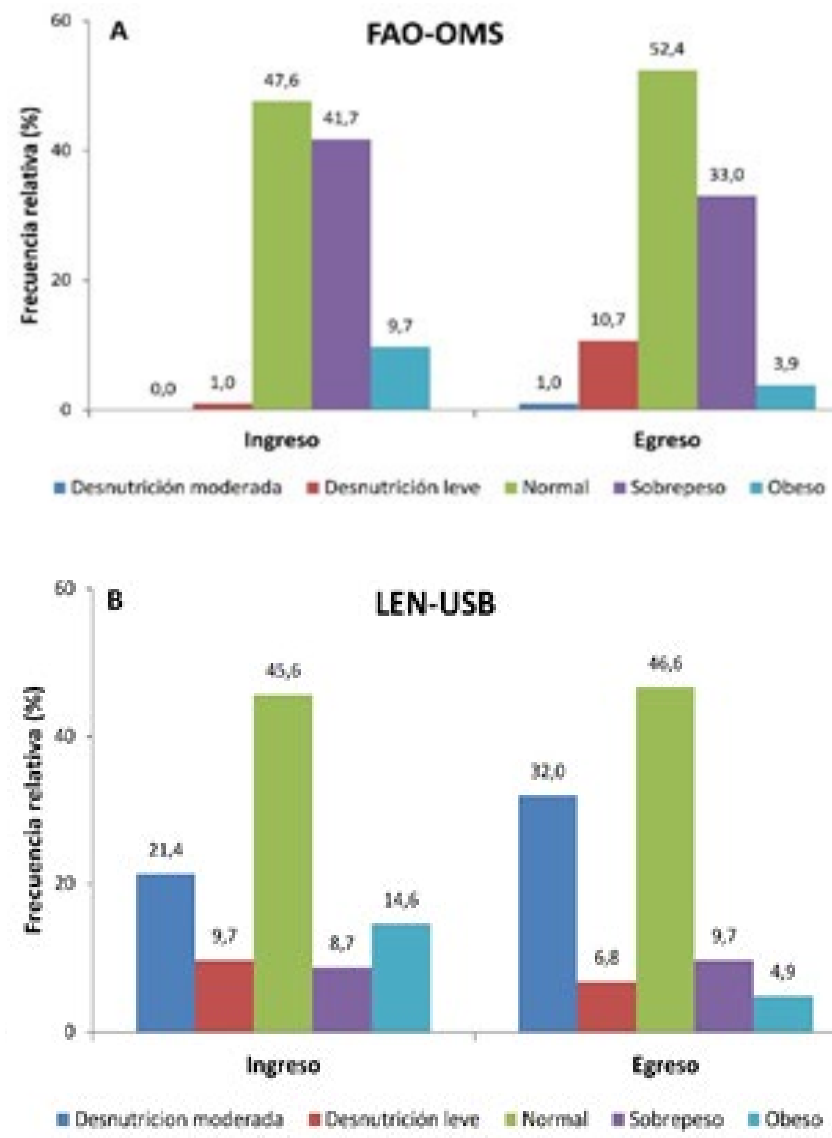

Fig. 3. Diagnóstico nutricional basado en IMC $(\mathrm{kg} / \mathrm{m} 2)$. A) Criterio de la FAO-OMS, B) Criterio del LEN-USB.
La prueba de rangos con signo de Wilcoxon indicó que hubo cambio estadísticamente significativo entre las categorías de la valoración nutricional al egreso con respecto al ingreso, tanto para el criterio FAO-OMS (SRank $\mathrm{f}_{(-)}=-528$; SRank $_{(+)}=0$; $p<0,0001)$ como para el criterio LEN-USB (SRank $k_{(-)}=-528$; SRank $\left._{(+)}=0 ; p<0,0001\right)$.

El coeficiente $\mathrm{K}$ de Cohen indicó que si bien hubo concordancia estadísticamente significativa en la valoración nutricional según los criterios por FAO-OMS y LEN-USB al ingreso, $(k=0,150 ; p<0,001)$ y al egreso $(k=0,173 ; p<0,001)$, en ambos casos la concordancia fue muy pobre según el criterio propuesto por López y Pita (20).

Al combinar IMC (LEN-USB) con \%PP para realizar diagnóstico nutricional, se aprecia al Ingreso: $14,6 \%$ Obesos grado II, $7,8 \%$ sobrepesados, $35,9 \%$ eutróficos, $0.9 \%$ soprepesado con desnutrición aguda, $10 \%$ eutrófico con desnutrición aguda y $31 \%$ desnutridos; mientras que el de egreso: $6,8 \%$ eutróficos, $39,8 \%$ eutróficos con desnutrición aguda, 9,7\% sobrepesados con desnutrición aguda, 4,8\% obesos con desnutrición aguda, (la pérdida de peso en estos pacientes fue de moderada a severa) y $38,8 \%$ desnutridos (Fig. 4).

Si bien la asociación entre el criterio LEN-USB y el \%PP no fue estadísticamente significativa $(x 2=11,32 ; p=0,470)$, sí permitió evidenciar la presencia de desnutrición hospitalaria al egreso, con una prevalencia de 92,2\% (95/103), la cual afectó a todos los pacientes por igual, independientemente de su estado nutricional inicial.

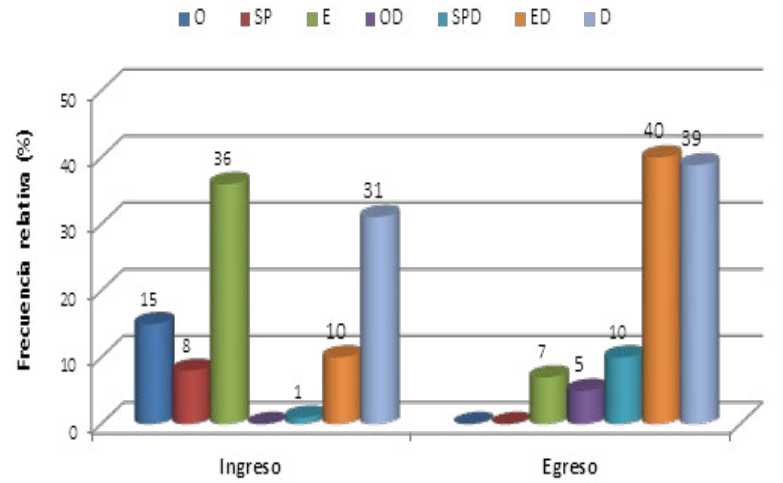

Fig. 4. Diagnóstico nutricional por la variable antropométrica IMC (LEN-USB) combinada con porcentaje pérdida de peso. $\mathrm{O}=$ obeso, $\mathrm{SP}=$ sobrepesado, $\mathrm{E}=$ eutrófico, $\mathrm{SPD}=\mathrm{SP}$ condesnutrición, $E D=$ eutrófico con desnutrición, $D=$ desnutrido.

\section{DISCUSIÓN}

La muestra se caracterizó por una amplia variabilidad en cuanto al grupo etario, la cual se corresponde con el comportamiento típico de una población viva, representando además, patologías diversas, no circunscriptas a una especialidad específica (21).

EI IMC es la relación entre peso y talla y no discrimina composición corporal (masa magra y grasa), es un método de evaluación nutricional antropométrica ampliamente utilizado, pero pierde capacidad para detectar cambios agudos. Comparando el diagnóstico nutricional por IMC 
utilizando los puntos de corte de la FAO/OMS y de LENUSB; se observó que ambos detectaron paciente con algún grado de desnutrición tanto al ingreso (1\% vs. $31,1 \%$ ) como al egreso (12\% vs. $38,8 \%)$, siendo mayor el porcentaje de desnutrición utilizando los puntos de corte de LEN-USB, (Fig. 3). Esta diferencia se debe a que los puntos de corte de LEN-USB están corregidos por sexo, edad y contextura, variables biomédicas que afectan la composición corporal, (el género femenino tiene más masa grasa, la tasa metabólica basal después de los 40 años se ralentiza), lo que permite detectar individuos con diferentes grados de bajo peso, particularmente en el paciente adulto mayor y anciano; su desventaja, es que requiere uso de gráficas, lo que puede ser complejo pero no difícil, para la práctica médica diaria $(1,15)$.

Ambos métodos no fueron capaces de diagnosticar la progresión de la desnutrición del paciente durante la estancia hospitalaria, aun presentando disminución de peso, en vista de que se mantuvieron dentro del mismo percentil durante la hospitalización, similar a lo descrito por Pérez-Flores et al. (12), Achaui (22), García et al. (23) y en contraposición a Álvarez-Hernández et al. (24). En una evaluación inicial se puede utilizar el IMC sin corregir por contextura, edad y sexo.

Se apreció que talla, peso usual, de ingreso y egreso fueron menores en las mujeres (25), (Fig. 1). El \%PP, es el cambio de peso relacionando con un referente, generalmente usual o actual, detectando pérdida aguda de peso, siendo el indicador más sensible de desnutrición aguda en el paciente hospitalizado, asociada generalmente a situaciones de estrés (infecciones, traumatismos, quemaduras, cirugías) con disminución de la masa grasa y masa magra, dando la mala impresión de buen estado nutricional. La pérdida involuntaria de peso superior al $10 \%$ en los últimos seis meses o el rápido desarrollo de la misma son signos clásicos de desnutrición (26-28). Hay que relacionar el \%PP en función del tiempo para valorar la gravedad de la misma, considerándose severa, pérdidas $>2 \%$ en 1 semana o $>20 \%$ en 6 meses $(16,19,29-31)$.

El \%PP al ingreso (usual y actual) fue mayor en las mujeres $(\bar{x}=-5,09 \pm 8,56)$ que en los hombres $(\bar{x}=-3,04 \pm 8,05)$ y más acentuada en los pacientes de la tercera edad, todos del departamento de medicina; mientras que los de traumatología y maxilofacial no refirieron pérdida de peso (Tabla 3), lo que lleva a pensar que el estrés ocasionado por la enfermedad de base es causante del cambio de peso en estos pacientes antes de ingresar (32), resultando en un factor de riesgo de morbimortalidad durante la hospitalización, de allí la importancia de no solamente tallar y pesar (33) al paciente a su ingreso, sino también determinar el \%PP y la severidad de la misma, tanto al ingresar como durante la hospitalización (Fig. 2).

Durante la hospitalización el 96\% de los pacientes presentó \%PP (severa (88\%) siendo más acentuada en los pacientes quirúrgicos (Tabla 3), cifras superiores a las reportadas por Waitzberg et al. (30), Fuchs et al. (34), Leandro-Merhi y Braga de Aquino (35) y Loreto (36). En términos relativos, las mujeres perdieron más peso por día que los hombres y las diferencias pequeñas entre las variables fueron más acentuadas en ellas (Tabla 2), en concordancia con Veramendi-Espinoza et al. (32).

El diagnóstico nutricional no es estático ni involucra un solo parámetro (37), en el presente estudio el IMC detectó desnutrición, pero al combinarlo con \%PP la prevalencia de desnutrición tanto al ingreso (42\%) como al egreso $(93 \%)$ fue significativamente mayor $(38,39)$.

Al ser el \%PP significativo, el paciente puede diagnosticarse con desnutrición aguda (leve, moderada, severa) independientemente del valor IMC obtenido, en este estudio se evidenció pacientes que ingresaron con sobrepeso por IMC y que egresaron eutróficos $u$ obesos grado II que egresaron sobrepesados o eutróficos, debido al \%PP severo que presentaron. La PP no intencional en función del tiempo se correlaciona de manera estrecha con el grado de riesgo y es el indicador más sensible de desnutrición aguda durante la hospitalización $(15,30,40)$ por lo que se considera una variable muy útil para detectar desnutrición en el paciente hospitalizado $(8,15,37,39,40)$.

La prevalencia de desnutrición hospitalaria observada en la presente investigación utilizando las variables antropométricas IMC (discriminada por edad y sexo) y \%PP durante la hospitalización, es superior a las reportada en otros países latinoamericanos $(32,41,42)$. La gran variabilidad en la prevalencia de desnutrición descrita en la literatura puede estar en relación con las características del hospital, la patología del paciente, la metodología y técnicas empleadas para su detección (7) y, en muchos casos, a una infravaloración de su frecuencia. Otras causas relacionadas con su elevada prevalencia son el fallo en la detección de las necesidades nutricionales y el escaso énfasis concedido al estado nutricional en la historia clínica $(38,43)$.

La desnutrición del paciente hospitalizado tiene relación con los días de estancia hospitalaria $(35,42,44)$, evidenciándose en el presente con significancia estadística $(p=0,0001)$ (Fig. 1). Durante la hospitalización el paciente se enfrenta a múltiples situaciones que repercuten en su condición nutricional, que no solamente se refiere a la carencia calórico-proteica y el aumento de la demanda metabólica de la enfermedad, sino también, procedimientos terapéuticos y diagnósticos que ameritan ayuno (muchas veces repetitivos o prolongados), administración de drogas, interrupción de alimentación por revistas médicas o tomas de muestras, que son mantenidas durante la estancia del paciente, estos son factores independientes de la enfermedad que influyen en la desnutrición proteico-calórica $(43,45)$.

En lo que refiere a la alimentación no hubo un efecto positivo en el tipo de dieta indicada, ya que el $92 \%$ de los pacientes mostró \%PP $(p=0,003)$ (moderada 0 severa) a su egreso indistintamente de la dieta indicada. Este resultado probablemente sea consecuencia de alimentación inadecuada en calidad o cantidad para proporcionar los nutrientes y calorías requeridos en base a su patología. Este es otro de los factores independientes de la enfermedad asociados a la desnutrición hospitalaria. En el presente estudio se observó que indistintamente del estado nutricional con el que ingresaron los pacientes, a ninguno se le indicó interconsulta con el servicio de

\section{Salus Revista de la facultad de ciencias de la Salud. Universidad de Carabobo. Mayo/agosto 2021 Vol. 25 № 2}


nutrición, no se calculó el aporte calórico requerido según las demandas enérgicas, así como tampoco se indicó suplementos alimenticios, condicionando una inadecuada prescripción dietética, situación que supera lo referido por Vasquez et al. (13) y Giraldo et al. (40). Dada la relevancia del problema de la desnutrición, tanto por su prevalencia, como por las consecuencias clínicas y económicas que conlleva, diversos organismos internacionales y sociedades científicas han remarcado la necesidad de un método de tamizaje o cribado validado, fiable, reproducible, práctico y coordinado con protocolos específicos de actuación, no utilizados en la presente investigación. Existen métodos de cribado clínico, automatizado y mixto. La mayoría de los métodos de cribado clínicos suelen incluir datos subjetivos y objetivos (peso, talla, cambios en el peso, cambios en la ingesta, comorbilidades, entre otras) $(32,46,47)$.

A pesar de que el aumento de la calidad de vida en la población representa uno de los principales triunfos médicos y sociales, la desnutrición hospitalaria sigue siendo un trastorno subestimado, no diagnosticado, ni tratado, que afecta adversamente tanto al individuo como a la sociedad.

\section{CONCLUSIONES}

La prevalencia de desnutrición hospitalaria utilizando IMC (discriminado por sexo y edad) asociado con porcentaje de pérdida de peso fue alta (93\%), independientemente del grupo etario y patología. El $95 \%$ de los pacientes presentaron pérdida de peso durante la hospitalización, siendo catalogada severa en el $88 \%$ de los casos. EI IMC en el paciente hospitalizado pierde capacidad para detectar desnutrición hospitalaria si es utilizado como único indicador antropométrico. El porcentaje de pérdida de peso en función del tiempo es la variable antropométrica que en el paciente hospitalizado permite detectar desnutrición aguda. La diferencia de peso observada al egreso fue más acentuada en el paciente quirúrgico mientras que al ingreso fue más evidente en los pacientes del departamento de medicina, esto evidencia que el estrés ocasionado por la enfermedad o la intervención quirúrgica es uno de los factores asociados a la desnutrición hospitalaria. La tasa de pérdida de peso diario, fue más rápida en pacientes de sexo femenino.

\section{REFERENCIAS BIBLIOGRÁFICAS}

1. Mora RJF. Malnutrición: Consecuencias orgánicas y funcionales. En: Mora R. Soporte Nutricional Especial. 3ra edición. Ed. Panamericana. 2000; pp 68-71.

2. Vesga AL, Gamboa EM. Riesgo de malnutrición asociado a baja ingesta alimentaria, estancia hospitalaria prolongada y reingreso en un hospital de alto nivel de complejidad en Colombia. Nutr Hosp 2015; 32(3):1308-14. Doi: https://dx.doi. org/10.3305/nh.2015.32.3.9416

3. Savino P. Desnutrición hospitalaria: grupos de soporte metabólico y nutricional. Rev Colomb Cir 2012; 27(1):46-54.

4. OMS. Temas de salud: Nutrición. [Serie en internet]. 2016]. Disponible en: https://www.who.int/health-topics/nutrition

5. FAO, OMS. Segunda Conferencia Internacional sobre Nutrición. Notas de las copresidencias de acompañamiento de los documentos finales de la conferencia. Roma 2014; 2:2-1. Fao.org/3/mm222S/mm222S.pdf.
6. Bistrian BR, Blackburn GL, Vitale J, Cochran D, Naylor. Prevalence of malnutrition in general medical patients. JAMA 1976; 235(1):1567-70. Doi: https://dx.doi.org/10.1001/ jama.1976.03260410023017

7. Salvador-Monferrer L, Fernández-Olea MS, Murillo-Sanchis J. Desnutrición y factores que influyen en la ingesta de alimentos en pacientes hospitalizados: una revisión. Nutr Clin Diet Hosp 2014; 34(3): 80-91. Doi: https://dx.doi.org/10.12873/343salva dormonferrer

8. Gomes-Beghetto M, Koglin G, Daniel de Mello E. Influence of the assessment method on the prevalence of hospital malnutrition: a comparison between two periods. Nutr Hosp 2010; 25(5): 774-80. Doi: https://dx.doi.org/10.3305/ nh.2010.25.5.4511

9. Kamath SK, Lawler M, Smith AE, Kalat T, Olson R. Hospital malnutrition: a 33-hospital screening study. J Am Diet Assoc 1986; 86(1):203-6. PMID: 3080513

10. Monti GR. Desnutrición hospitalaria: una patología subdiagnosticada. Rev Asoc Med Arg 2008; 121(4): 28-31.

11. Ortiz J. Evaluación del estado nutricional del adulto mayor institucionalizado a través de la escala mini nutritional assessment (MNA). [Tesis especialista]. Universidad de Carabobo. Facultad de Ciencias de la Salud; 2013.

12. Pérez-Flores JE, Chávez-Tostado $M$, Larios-del-Toro YE, García-Rentería J, Rendón-Félix J, Salazar-Parra M, et al. Evaluación del estado nutricional al ingreso hospitalario y su asociación con la morbilidad y mortalidad en pacientes mexicanos. Nutr Hosp 2016; 33(4):872-8. Doi: https://dx.doi. org/10.20960/nh.386

13. Vázquez A, Flores $Q$, Chirinos J. Desnutrición hospitalaria en un hospital privado de la ciudad de México. Lo que hay después del tamizaje. Rev Nutri Clin Metab 2020. 3(1):59-65. https://doi.org/10.35454/rncm.v3n1.024

14. Botina DC, Ayala VA, Paz IC, Limas LA, Mafla AC. Estado nutricional en pacientes hospitalizados del Hospital Universitario Departamento Nariño. Rev Univ Ind Santander Salud 2013. 45(3):5-17.

15. Herrera HA, Hernández R, Rodríguez N. Manual de procedimientos y técnicas antropométricas. Centro de Investigaciones Nutricionales Antropológicas y de salud. USB 2007; pp 1-45.

16. FAO/OMS. Physical Status: the use and interpretation of anthropometry. Geneva: WHO Expert Committee; 1995; 1-158. Disponible en: https://apps.who.int/

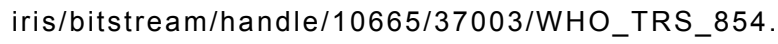
pdf? sequence $=1$ \&isAllowed $=y$

17. FELANPE. Curso Interdisciplinario de Nutrición Clínica (Manual). XI Congreso de la Federación Latinoamericana de Terapia Nutricional, Nutrición Clínica y Metabolismo. CancúnMéxico 2008; pp:1-76.

18. Frisancho AR. Anthropometric standards for the assessment of growth and nutritional status. The University of Michigan Press. Ann Arbor-MI 1990. 189 p. Doi: https://doi.org/10.1002/ ajpa. 1330840116 
19. Blackburn GL, Bistrian BR, Maini BS, Schlamm HT, Smith MF. Nutritional and metabolic assessment of the hospitalized patient. JPEN J Parenter Enteral Nutr 1977; 1(1):11-22. Doi: https://doi.org/10.1177/014860717700100101

20. López I, Pita S. Medidas de concordancia: el índice de Kappa. Cad Aten Primaria 1999, 6(1):169-171.

21. Fernández JA. Índices de relación peso-talla como indicadores de masa muscular en mujeres adultas. Rev Cuba Alim Nutr 2002; 16(1):114-8.

22. Achahui C. Prevalencia de desnutrición en pacientes diabéticos hospitalizados en el servicio de endocrinología del HNGAI [Tesis]. Lima, Perú: Universidad Nacional Mayor de San Marcos. Facultad de Medicina Humana; 2004. https:// cybertesis.unmmsm.edu.pe/handle/20.500.12674/2003

23. García C, Muñoz C, Demelo P, Cerezo E, Echevarría R, Fraga J, et al. Parámetros antropométricos y analíticos en la valoración del estado nutricional de los pacientes. Rev Clin Esp 2014; 214(Espec Congr):1119.

24. Álvarez-Hernández $J$, Planas $M$, León-Sanz $M$, García $A$, Celaya-Pérez S, García-Lorda P, et al. Prevalence and costs of malnutrition in hospitalized patients; the PREDyCES $® S t u d y$. Nutr Hosp 2012; 27(4):1049-59. Doi: https://dx.doi.org/10.3305/ nh.2012.27.4.5986

25. Matarese L. Valoración metabólica y nutricional de pacientes hospitalizados. En Patiño JF. Metabolismo, nutrición y shock. 4ta edición Ed. Panamericana. 2006: pp 155-163

26. Villamayor L, Llimera G, Jorge V, González C, Iniesta C, Mira MC, et al. Valoración nutricional al ingreso hospitalario: iniciación al estudio entre distintas metodologías. Nutr Hosp 2006; 21(2):165-72.

27. Ravasco $\mathrm{P}$, Anderson $\mathrm{H}$, Mardones F. Métodos de valoración del estado Nutricional. Nutr Hosp 2010; 25(suppl 3):57-66.

28. Espinosa A, Martínez C, Barreto J, Santana S. Esquema para la evaluación antropométrica del paciente hospitalizado. Rev Cuba Alim Nutr 2007; 17(1):72-89.

29. Elia M. Hunger disease. Clin Nutr 2000; 19(6):379-86. Doi: https://doi.org/10.1054/clnu.2000.0157

30. Waitzberg D, Ravacci G, Raslan M. Desnutrición hospitalaria. Nutr Hosp 2011; 26(2):254-64. Doi: https://doi.org/10.3305/ nh.211.26.2.5070

31. Müller $\mathrm{O}$, Krawinkel $\mathrm{M}$. Malnutrition and health in developing countries. CMAJ. 2011; 173(3):279-86. Doi: https://doi. org/10.1503/cmaj.050342

32. Veramendi-Espinoza LE, Zafra-Tanaka JH, Salazar-Savedra $\mathrm{O}$, Basilio-Flores JE, Millones-Sánchez E, Pérez-Casquino $\mathrm{GA}$, et al. Prevalencia y factores asociados a desnutrición hospitalaria en un hospital general; Perú, 2012. Nutr Hosp 2013; 28(4):1236-43. Doi: https://dx.doi.org/10.3305/ nh.2013.28.4.6390

33. Monterrey P, Porrata C. Procedimiento gráfico para la evaluación del estado nutricional en adultos según el índice de masa corporal. Rev Cuba Alim Nutr 2001. 15(1):62-7.

34. Fuchs V, Mostkoff D, Gutiérrez G, Amancio O. Estado nutricio en pacientes internados en un hospital público de la ciudad de México. Nutr Hosp 2008; 23(3):294-303.
35. Leandro-Merhi VA, Braga de Aquino JL. Nutritional status and length of hospital stay for surgical patients. Nutr Hosp 2010; 25(3):468-9.

36. Loreto $\mathrm{S}$. Valoración nutricional en pacientes hospitalizados en el servicio de medicina interna. [Tesis especialista]. Universidad de Carabobo; 2015. https://riuc.bc.uc.edu.ve/ bitstream/123456789/4931/1/sloreto.pdf.

37. Ulíbarri JI. La desnutrición clínica en 2014; patogenia, detección precoz y consecuencias; desnutrición y trofopatía. Nutr Hosp 2014; 29(4):785-96. Doi: https://dx.doi.org/10.3305/ nh.2014.29.4.7272

38. Ulíbarri JI, Lobo G, Pérez AJ. Desnutrición clínica y riesgo nutricional en 2015. Nutr Clin Med 2015; 9(3):231-54.

39. González A, Mancha A, Rodríguez FJ, de Ulibarri JI, Culebras J. The use of biochemical and immunological parameters in nutritional screening and assessment. Nutr Hosp 2011; 26(3):594-601. Doi: https://doi.org/10.1590/s021216112011000300024

40. Giraldo NA, Múnera NE, Marrugo V, Piñeres LM. Prevalencia de malnutrición y evaluación de la prescripción dietética en pacientes adultos hospitalizados en una institución pública de alta complejidad. Perspect Nutr Hum 2007; 9(1):37-47.

41. González L, Coloma R, Ascorbe P, Indo O, Rodríguez B, Martínez MJ. Estado actual del grado de desnutrición en los pacientes hospitalizados de la Comunidad de La Rioja. Nutr Hosp 2001; 16(1):7-13.

42. Waitzberg DL, Caiaffa WT, Correia MI. Hospital malnutrition: the Brazilian national survey (IBRANUTRI): a study of 4000 patients. Nutrition 2001; 17(7-8):573-80. Doi: https://doi. org/10.1016/s0899-9007(01)00573-1

43. Butterworth CE Jr. The skeleton in the hospital closet. Nutrition 1994; 10(5):442. PMID: 7819665.

44. Kyle UG, Coss-Bu JA. Nutritional assessment and length of hospital stay. CMAJ 2010; 108(17):1831-2. Doi: https://doi. org/10.1503/cmaj.101256

45. Santana S. Comentario al artículo El esqueleto en la taquilla del hospital. Nutr Hosp 2005; 20(4):297-307.

46. Detsky AS, McLaughlin JR, Baker JP, Johnston N, Whittaker $\mathrm{S}$, Mendelson RA, et al. What is subjective global assessment of nutritional status? JPEN 1987; 11(1):8-13. Doi: https://doi. org $/ 10.1177 / 014860718701100108$

47. Ulibarri JI, Burgos R, Lobo G, Martínez MA, Planas M, Pérez A, et al. Recomendaciones sobre la evaluación del riesgo de desnutrición en los pacientes hospitalizados. Nutr Hosp 2009; 24(4):467-72 